\title{
Analysis of Consensus-based Islanded Microgrids Subject to Unexpected Electrical and Communication Partitions
}

\author{
Carlos Xavier Rosero, Manel Velasco, Pau Martí, Antonio Camacho, Jaume Miret, Member, IEEE, \\ and Miguel Castilla
}

\begin{abstract}
Microgrids are power systems consisting of an electrical network composed by distributed loads and generation units that may include a communication network for improved operation. The considered microgrid in islanded mode is driven by voltage source inverters implementing decentralized droop control for active power sharing together with a communicationbased consensus algorithm for frequency regulation. This paper analyses the microgrid performance subject to network failures that provoke network partitions. It is considered that the electrical partition leads to several sub-microgrids working in parallel where the power demand can be always guaranteed by the generation units, and the communication partition leads to several consensus algorithms also working in parallel. The double partitioning is analyzed through a closed-loop system model derived using the power flow equations that includes the electrical and communication connectivity. Analytical expressions for the steady-state values for both frequency and active power depending on the partitioning are derived. Selected experimental results on a low-scale laboratory microgrid illustrate the (undesirable) impact that unexpected partitions have in system performance.
\end{abstract}

Index Terms-Microgrids, islanded mode, power sharing, frequency regulation, droop control, consensus control, graph theory, partitioning.

\section{INTRODUCTION}

Microgrids (MGs) are small-scale power systems containing storage elements, loads and distributed generators (DG) that are interfaced with the electric network via power electronic inverters [1]. When a MG is in islanded mode, its dynamics are no longer dominated by the main grid, and inverters acting as voltage source inverters (VSI) must take coordinated actions to ensure synchronization, voltage regulation, power balance and load sharing [2], [3]. Diverse control strategies addressing these challenges have been proposed, often organized according to a standardized three-level control architecture [4] that operates supported by a communication network. The scope of this paper is bounded to islanded MGs driven by VSI implementing decentralized droop control [5] for active

This work has been supported by ELAC2014/ESE0034 from the European Union and its linked Spanish national project PCIN-2015-001. We also appreciate the support from the Ministry of Economy and Competitiveness of Spain and the European Regional Development Fund (FEDER) under project ENE2015-64087-C2-1-R

C.X. Rosero, M. Velasco, P. Martí and A. Camacho are with the Automatic Control Department, Technical University of Catalonia, Pau Gargallo 5, 08028 Barcelona, Spain, email: cxrosero@utn.edu.ec, $\{$ manel.velasco,pau.marti,antonio.camcho.santiago\}@upc.edu

J. Miret and M. Castilla are with the Electronic Engineering Department, Technical University of Catalonia, Av. Victor Balaguer s/n, 08800 Vilanova la Geltrú, Spain, email:\{jaume.miret,miquel.castilla\}@upc.edu power sharing together with a communication-based consensus algorithm for frequency regulation [6].

Apart from the known impact that natural disasters have in power systems, e.g. [7], the use of communication networks also impacts power systems, e.g. [8]. Hence, the interdependency between the electrical network and the communication network makes the problems of reliability, operation, and security more complex than in the traditional power grid. Identifying, understanding, and analyzing such interdependencies are significant challenges [9]. There are numerous studies on interdependencies in combined power and communication systems (see a recent state-of-the-art review in [10]). Many of them have provided conceptual insights into critical interdependencies, and/or statistical tools for risk assessment, e.g. [11], [12]. In addition, these studies usually apply on larger scale power systems than autonomous MGs, with different structural properties such as communication network redundancy [13].

Interdependencies and reliability issues in MGs have not been widely discussed in the literature and the study of both electrical and communication partitions becomes relevant to ensure their correct and safe operation. In addition it provides insight into the trade-offs associated with this technology where power and communications networks may be tightly coupled like in the case treated in this paper. The analysis discovers the impact of both network's failures have in terms of MG active power sharing and frequency regulation.

The analytical tools used in this paper that are based on graph theory are not new. MGs control approaches where the electrical and/or the communication network are modeled as graphs can be found for example in [6], [14]-[27]. But only a few of them modeled the MG in closed-loop form in terms of both Laplacian matrices. The focus on the zero eigenvalue analysis of the Laplacian matrices applied in this paper has been also used for example in [27] (and references therein) in the context of small-disturbance stability analysis of power systems in terms of critical lines. However, the scenario covered in this paper where each partitioned graph transforms into several isolated sub-graphs and its relation to the zero eigenvalues of the electrical and communication Laplacian matrices has not been previously reported.

\section{A. Motivating example}

Figure 1 illustrates the case of a simulated MG composed by 16 VSIs, grouped in four sub-MGs, $s M G_{1,2,3,4}$ (labeled by 


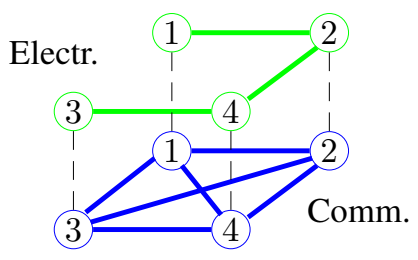

(a) Standard operation

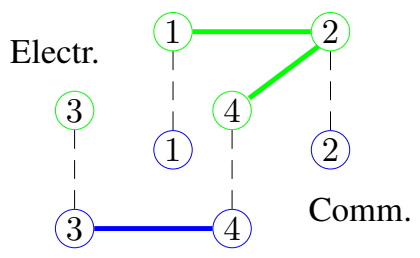

(b) After a failure
Fig. 1: 16-VSI MG electrical and communication connectivity.

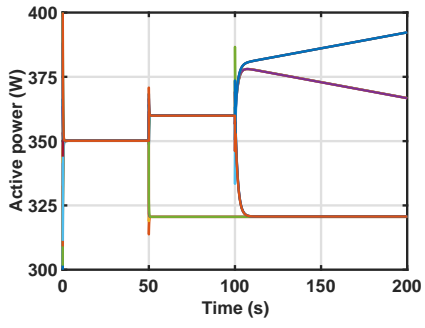

(a) Complete simulation

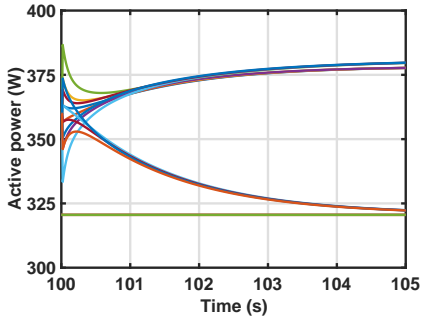

(b) Detail of the simulation
Fig. 2: 16-VSI MG injected active power after two failures.

1, 2, 3, and 4), each one including four generators (connected electronically and by communications) and a main load that is different for each $s M G_{i}$. Each $s M G$ is similar to the scheme shown in Figure 4 that will be further explained in the results section. The $4 s M G$ s are electronically connected through transmissions lines (in green), and also connected using a communication infrastructure (in blue). Sub-figure 1(a) corresponds to the connectivity in standard operation. Subfigure 1(b) describes the connectivity after two failures: an electrical failure that disconnects $s M G_{3}$ from the rest, and after a communication outage that leads to a situation where communications are only possible between $s M G_{3}$ and $s M G_{4}$.

Figure 2 shows the 16-VSI injected active power when the electrical failure occurs at time $t=50 \mathrm{~s}$ and the communication outage occurs at time $t=100 \mathrm{~s}$, giving the complete $200 \mathrm{~s}$ simulation in Sub-figure 2(a), and a detailed zoom from $t=100 \mathrm{~s}$ to $t=105 \mathrm{~s}$ in Sub-figure 2(b). The starting point corresponds to the scenario where no failures occur and all VSIs share the active load demand, delivering $350 \mathrm{~W}$ each. When the electrical failure occurs at time $t=50 \mathrm{~s}$, the 4 VSIs belonging to $s M G_{3}$ work isolated form the rest, sharing their local load demand and lowering the injected power to $320.5 \mathrm{~W}$ each, while the rest of 12 VSIs belonging to $s M G_{1,2,4}$ are still electrically connected and share their local loads demands by increasing the injected power up to $360 \mathrm{~W}$ each. Then, when the communication outage occurs at time $t=100 \mathrm{~s}$, two different dynamics can be observed. First, the 4 VSIs belonging to $s M G_{4}$, that were injecting $360 \mathrm{~W}$ each, reduce their injection to the same level of the 4 VSIs belonging to $s M G_{3}$, thus injecting $320.5 \mathrm{~W}$ each, because the communication link between $s M G_{3}$ and $s M G_{4}$ is still alive. Secondly, the 4 VSIs belonging to $s M G_{1}$ and the 4 VSIs belonging to $s M G_{2}$, that were injecting $360 \mathrm{~W}$ each, start injecting the same amount of active power but the dynamics have different slope, leading to a dangerous unstable operation.

\section{B. Paper contributions and structure}

The contribution of this paper is to provide an analytical framework for performance analysis that permits to predict and analyze the effects that electrical and communication failures have in terms of active power sharing and frequency regulation. The analysis is based on a closed-loop model able to capture the networks partitions, thus permitting to analyze their impact in the MG performance. Numerical examples and selected laboratory experiments are used to describe the behavior of the MG under different partition scenarios, which permits identifying serious risks. It must be noted that the assessment of secondary voltage control of $\mathrm{MG}$ operation under failures is not treated in this paper because the existing variety of control objectives deserves a complete analysis that does not fit in this paper.

The rest of the paper is structured as follows. Section II introduces the closed-loop model. Section III provides the theoretical analysis and Section IV presents a numerical example and selected experiments. Section V concludes the paper.

\section{MODELING APPROACH}

The MG electrical network is a generic connected grid where loads are modeled by constant impedances. Although future work will consider more rich configurations (e.g. nonlinear and time-varying loads), keeping a simplified model helps gaining understanding and reaching results that will permit dealing with more complex MGs. A Kron reduction is performed which allows obtaining a lower dimensional dynamically-equivalent model described by ordinary differential equations [28], [29]. The reduced network is modeled as a connected undirected graph $\mathcal{G}_{e}=\left\{\mathcal{N}_{e}, \mathcal{E}_{e}\right\}$ where the $n_{e}$ nodes $\mathcal{N}_{e}$ represent DGs interfaced with VSIs and edges $\mathcal{E}_{e} \subseteq \mathcal{N}_{e} \times \mathcal{N}_{e}$ represent the power lines. Nodes are characterized by a phase angle $\theta_{i}$ and a voltage amplitude $v_{i}$. Edges represent line admittances between nodes $i$ and $j$ as $y_{i j}=g_{i j}+\mathrm{j} b_{i j} \in \mathbb{C}^{+}$, where $g_{i j} \in \mathbb{R}^{+}$is the conductance and $b_{i j} \in \mathbb{R}^{+}$is the susceptance. The electrical network is represented by the symmetric bus admittance matrix $Y \in \mathbb{C}^{n_{e}} \times \mathbb{C}^{n_{e}}$, where the off-diagonal elements are $Y_{i j}=Y_{j i}=-y_{i j}$ for each edge $\{i, j\} \in \mathcal{E}_{e}$, and the diagonal elements are given by $Y_{i i}=\sum_{i=1}^{n_{e}} y_{i j}$. It is assumed that the reduced MG is connected.

The active power injected by each $i^{t h}$ node is described as

$$
p_{i}(t)=v^{2} \sum_{j=1}^{n} g_{i j}+v^{2} \sum_{j=1}^{n} b_{i j}\left(\theta_{i}(t)-\theta_{j}(t)\right)
$$

assuming that nodes phase angles and voltages are similar, as usually assumed in power systems modeling, e.g. [30], and also in MG modeling, e.g. [31]. Hence, the accuracy of the paper analysis improves whenever this assumptions hold. By considering the matrix $G \in \mathbb{R}^{n \times n}$ formed by the line conductances whose entries are given by $G_{i j}=g_{i j}$, denoting the set of phase angles by $\Theta(t)=\left[\theta_{1}(t) \cdots \theta_{n}(t)\right]^{T}$, and the set of active powers by $P(t)=\left[p_{1}(t) \cdots p_{n}(t)\right]^{T}$, the active power of the Kron-reduced network (1) becomes

$$
P(t)=v^{2} G \mathbf{1}_{n \times 1}+v^{2} B \Theta(t)
$$


where $\mathbf{1}_{n \times 1} \in \mathbb{R}^{n \times 1}$ denotes a vector of ones, and $B \in \mathbb{R}^{n \times n}$ is the Laplacian matrix of the power system given by

$$
B=\left[\begin{array}{cccc}
\sum_{\substack{j=1 \\
j \neq 1}}^{n} b_{1 j} & -b_{12} & \cdots & -b_{1 n} \\
-b_{21} & \sum_{\substack{j=1 \\
j \neq 2}}^{n} b_{2 j} & \cdots & -b_{2 n} \\
\vdots & \vdots & \cdots & \vdots \\
-b_{n 1} & -b_{n 2} & \cdots & \sum_{\substack{j=1 \\
j \neq n}}^{n} b_{n j}
\end{array}\right]
$$

formed by the line susceptances.

The goal of the considered control is to set the nodes phases $\theta_{i}(t)$ to achieve power sharing while having the frequency at the desired set-point. Each node $i \in \mathcal{N}_{e}$ is modeled as a control algorithm implemented at each VSI driven by

$$
\begin{aligned}
\dot{\theta}_{i}(t) & =\omega_{i}(t) \\
\omega_{i}(t) & =\omega_{0 i}-m_{i} p_{i}(t)+\delta_{i}(t)+\varphi_{i}(t) \\
\delta_{i}(t) & =k_{i} \int_{0}^{t} \omega_{0 i}-\omega_{i}(t)+\frac{c_{i}}{n_{e}} \sum_{j=1}^{n_{e}} a_{i j}\left[\delta_{j}(t)-\delta_{i}(t)\right] \mathrm{d} t
\end{aligned}
$$

where each node phase (4) varies according to the droop control [5] (5) that includes a corrective term $\delta_{i}(t)$ for frequency restoration (6) inspired in the consensus control included in [6]. The droop control (5) includes a perturbation term $\varphi_{i}(t)$ modeling bounded uncertainties such as measurement errors or disturbances. Although not explicitly formalized in (5), the droop control used in the experimental set-up will be enabled with output virtual impedance to avoid controllability problems that otherwise may occur [32]. However, for the sake of simplicity, it is omitted in the theoretical development because its inclusion would not alter the obtained results. The correction term $\delta_{i}(t)$ in (6) is computed in part using the neighbors correction terms $\delta_{j}(t)$ that must be exchanged over a communication network.

The communication network can also be represented by a connected undirected graph $\mathcal{G}_{c}=\left\{\mathcal{N}_{c}, \mathcal{E}_{c}\right\}$ where the $n_{c}$ nodes $\mathcal{N}_{c}$ represent DGs interfaced with VSIs that implement (4)-(6), and edges $\mathcal{E}_{c} \subseteq \mathcal{N}_{c} \times \mathcal{N}_{c}$ represent communication links. Parameters $a_{i j}$ in the consensus control (6) form the adjacency matrix of $\mathcal{G}_{c}$ such that $a_{i j}=a_{j i}=1$ if nodes $i$ and $j$ can exchange their information and $a_{i j}=0$ otherwise. It is considered that nodes in the electrical and communication graph are the same, i.e. $\mathcal{N}_{e} \equiv \mathcal{N}_{c}$, hence $n_{e}=n_{c}=n$, which is the habitual situation in MGs, e.g. [6] or [21].

By denoting the set of VSI local frequencies by $\Omega(t)=$ $\left[\omega_{1}(t) \cdots \omega_{n}(t)\right]^{T}$, the set of desired frequencies by $\Omega_{0}=$ $\left[\omega_{01} \cdots \omega_{0 n}\right]^{T}$, the set of correction terms by $\Delta(t)=$ $\left[\delta_{1}(t) \cdots \delta_{n}(t)\right]^{T}$, the set of perturbations by $\Phi(t)=$ $\left[\varphi_{1}(t) \cdots \varphi_{n}(t)\right]^{T}$ and the diagonal matrices $M, K, C \in$ $\mathbb{R}^{n \times n}$ of droop gains $m_{i}$, consensus gains $k_{i}$ and $c_{i}$, respectively, the per-node control algorithm given in (4)-(6) can be compactly written as

$$
\begin{aligned}
& \dot{\Theta}(t)=\Omega(t) \\
& \Omega(t)=\Omega_{0}-M P(t)+\Delta(t)+\Phi(t) \\
& \Delta(t)=K \int_{0}^{t}\left(\Omega_{0}-\Omega(t)-\frac{1}{n} C L \Delta(t)\right) \mathrm{d} t
\end{aligned}
$$

where $L \in \mathbb{R}^{n \times n}$ in (9) is the Laplacian matrix of the communication graph $\mathcal{G}_{c}$ given by

$$
L=\left[\begin{array}{cccc}
\sum_{\substack{j=1 \\
j \neq 1}}^{n} a_{1 j} & -a_{12} & \cdots & -a_{1 n} \\
-a_{21} & \sum_{\substack{j=1 \\
j \neq 2}}^{n} a_{2 j} & \cdots & -a_{2 n} \\
\vdots & \vdots & \cdots & \vdots \\
-a_{n 1} & -a_{n 2} & \cdots & \sum_{\substack{j=1 \\
j \neq n}}^{n} a_{n j}
\end{array}\right]
$$

where $a_{i j}$ are the entries of the adjacency matrix of $\mathcal{G}_{c}$.

The goal of the control (7)-(9) is shaping the active power and frequency dynamics. The active power dynamics can be obtained by computing the derivative of (2) that leads to

$$
\dot{P}(t)=v^{2} B \dot{\Theta}(t) .
$$

By using (7) and (8), the active power variation (11) can be written as

$$
\dot{P}(t)=-v^{2} B M P(t)+v^{2} B \Delta(t)+v^{2} B \Omega_{0}+v^{2} B \Phi(t) .
$$

In addition, the frequency dynamics can be obtained by computing the derivative of (8) that leads to

$$
\dot{\Omega}(t)=-M \dot{P}(t)+\dot{\Delta}(t)+\dot{\Phi}(t)
$$

Noting that $\dot{\Delta}(t)$ is the derivative of (9), it follows that

$$
\dot{\Delta}(t)=K\left(\Omega_{0}-\Omega(t)-\frac{1}{n} C L \Delta(t)\right)
$$

which can be rewritten using (8) as

$$
\dot{\Delta}(t)=K M P(t)-K\left(\frac{1}{n} C L+\boldsymbol{I}_{n}\right) \Delta(t)-K \Phi(t)
$$

where $\boldsymbol{I}_{n} \in \mathbb{R}^{n \times n}$ is the identity matrix. The frequency dynamics (13) can be rewritten using (12) and (14) as

$$
\begin{aligned}
\dot{\Omega}(t)= & M^{2} v^{2} B P(t)-\left(M v^{2} B+K \frac{1}{n} C L\right) \Delta(t)-K \Omega(t) \\
& +\left(K-M v^{2} B\right) \Omega_{0}-M v^{2} B \Phi(t)+\dot{\Phi}(t)
\end{aligned}
$$

Gathering the equations of the power dynamics (12) and frequency dynamics (16), together with the correction term dynamics (15), the multiple-input/multiple output closed-loop dynamics are given by

$$
\left[\begin{array}{c}
\dot{P}(t) \\
\dot{\Delta}(t) \\
\dot{\Omega}(t)
\end{array}\right]=S\left[\begin{array}{l}
P(t) \\
\Delta(t) \\
\Omega(t)
\end{array}\right]+U \Omega_{0}+R\left[\begin{array}{l}
\Phi(t) \\
\dot{\Phi}(t)
\end{array}\right],
$$

where the closed-loop system matrix $S \in \mathbb{R}^{3 n \times 3 n}$, input matrix $U \in \mathbb{R}^{3 n \times n}$ and perturbation matrix $R \in \mathbb{R}^{3 n \times 2 n}$ are

$$
\begin{aligned}
S & =\left[\begin{array}{ccc}
-M v^{2} B & v^{2} B & \mathbf{0}_{n \times n} \\
K M & -K\left(\frac{1}{n} C L+\boldsymbol{I}_{n}\right) & \mathbf{0}_{n \times n} \\
M^{2} v^{2} B & -M v^{2} B-\frac{1}{n} K C L & -K
\end{array}\right] \\
U & =\left[\begin{array}{c}
v^{2} B \\
\mathbf{0}_{n \times n} \\
K-M v^{2} B
\end{array}\right], \quad R=\left[\begin{array}{cc}
v^{2} B & \mathbf{0}_{n \times n} \\
-K & \mathbf{0}_{n \times n} \\
-M v^{2} B & \boldsymbol{I}_{n}
\end{array}\right]
\end{aligned}
$$

being $\mathbf{0}_{n \times n} \in \mathbb{R}^{n \times n}$ a matrix of zeros. 
For the zero eigenvalue analysis presented next, it is important to note that for a given load the total power $P_{T}$ that is injected by the MG nodes is always the same, then

$$
\forall t, \quad \sum_{i=1}^{n} p_{i}(t)=P_{T} \quad \rightarrow \quad \sum_{i=1}^{n} \dot{p}_{i}(t)=0 .
$$

This means that one of the $\dot{p}_{i}(t)$ is linearly dependent of the others $\dot{p}_{j}(t), j=1, \ldots, n, j \neq i$, that is, 1 of the $3 n$ equations of (17) is linear dependent of the others. Looking at matrices $S$ (18) and $U$ (19), this dependency implies that $\operatorname{rank}(S)=$ $\operatorname{rank}(S \mid U) \leq 3 n-1$ and also implies that at least $S$ has one eigenvalue at zero that does not act as an integrator for the closed-loop dynamics. In fact, the zero eigenvalue would disappear with the minimal realization of (17). However, the non-minimal realization is kept because it provides a better intuitive description of the closed-loop dynamics in terms of meaningful physical variables. Note also that $\operatorname{rank}(S \mid R) \leq$ $3 n-1$, that is, the same structural property that exhibits the input matrix $U$ applies to the perturbation matrix $R$ in (17).

\section{PARTITION ANALYSIS}

For the partition analysis it is assumed that the control gains have been designed such that both control goals, active power sharing and frequency regulation, can be always reached. This holds for the scenario previous to a partition and for the new scenario appearing after a partition. Details of the design are out of the scope of this paper but suitable values for gains $M, C$ and $K$ can be obtained by solving a Linear Matrix Inequality (LMI) problem for all admissible (and possible) partitions. In addition, it is assumed that after any electrical partition, each sub-microgrid meets the local load demands with the local generation units. Otherwise, a cascading failure would occur leading to a collapse [33], [34]. Active power sharing (i.e., the power of the inverters in steady-state must be proportional to its power rating while guaranteeing the supply of the load) can be formulated as

$$
p_{i}(\infty)=\frac{P_{T}}{\sum_{j=1}^{n}\left(\frac{m_{i}}{m_{j}}\right)}
$$

where $p_{i}(\infty)$ is the active power provided by each inverter in steady-state, $P_{T}$ is the MG total load power, and $m_{i}$ and $m_{j}$ are the droop gains given in (5) related to the rated power of the inverters. Frequency regulation in steady-state in the $\mathrm{MG}$ can be formulated as

$$
\omega_{i}(\infty)=\omega_{0}
$$

where $\omega_{i}(\infty)$ is the local frequency of each inverter in steadystate.

From this scenario, it is analyzed how the eigenvalues of a stable closed-loop system vary due to the partitioning. Then, steady-state expressions for power and frequency are derived. Henceforth, the contribution of the perturbation term in the closed-loop dynamics (17) is explicitly omitted (i.e. $\Phi(t)=$ 0) to allow focusing on the effects that partitions have on the ideal dynamics. The inclusion of the perturbation term in the analysis is left for future work. However, whenever appropriate, it will be discussed for the sake of completeness.

\section{A. Characterization of the system eigenvalues}

Since the starting point for the analysis is that both the electrical and communication graphs are connected, it holds that their Laplacian matrices, $B(3)$ and $L(10)$, have a single 0 eigenvalue [35]. Whenever partition occurs, the original graphs are split into disconnected components that form electrical and/or communication sub-graphs that represent several sub-microgrids and/or several control algorithms. Then, the multiplicity of the 0 eigenvalue of the Laplacian matrices indicate the number of resulting disconnected components [35].

The closed-loop dynamics (17) characterized by the system matrix $S$ (18) and the input matrix $U$ (19) depend on the communication and electrical Laplacian matrices $L$ and $B$. In particular, $S$ depends on both $L$ and $B$ while $U$ depends only on $B$. And Theorems 1 and 2 (see Appendix A) permit concluding that any additional 0 eigenvalue in the Laplacian matrices $L$ or $B$ caused by a partition leads to a new 0 eigenvalue in $S$. Therefore, the dynamics the closed-loop system may be altered depending on the role of the additional 0 eigenvalue in the system matrix $S$.

When looking at the additional 0 eigenvalue in the system matrix $S$ caused by communication partition, it is worth noting that the input matrix $U$ does not depend on the communication Laplacian matrix $L$. Hence, this additional 0 eigenvalue becomes an integrator for the closed-loop dynamics (17) because matrix $S$ loses rank while matrix $S \mid U$ does not, thus maintaining the original set of $3 n-1$ linear independent equations. However, this is not the case for the additional 0 eigenvalue in the system matrix $S$ caused by an electrical partition because the input matrix $U$ does depend on the electrical Laplacian matrix $B$. In this case, the additional 0 eigenvalue does not become an integrator for the closed-loop dynamics (17) because both matrices $S$ and $S \mid U$ lose rank whenever $B$ loses rank, thus decrementing the set of linear independent equations.

In operational terms, whenever a communication partition occurs, the MG control algorithm transforms into two isolated control algorithms working in parallel on the same plant. And in terms of closed-loop dynamics, reminding that the closed loop system is multiple-input/multiple output, the additional 0 eigenvalue becomes an integrator of each input/output relation (from input $\omega_{0 i}$ to any of the outputs) in such a way that the system operation corresponds to $n$ integrators working in parallel. And this puts the overall $\mathrm{MG}$ into risk due to the destabilizing effects of integrators working in parallel [36], probably leading to large steady-state errors or even to unstable dynamics. Complementary, whenever an electrical partition occurs, the MG splits into several isolated MGs working in parallel and controlled by a single control algorithm. And in terms of closed-loop dynamics, the additional 0 eigenvalue does not have destabilizing effects identified for the case of the communication partition. However, power flows are strongly affected. Hence, after the occurrence of an electrical partition, since active power can not be physically transferred between the isolated MGs, cascading failures could occur if each submicrogrid supply-demand would not be able to reach the equilibrium. However, as outlined earlier, the adopted model 
assumes that the MG capacity has been dimensioned and control gains have been designed such that this equilibrium can be always reached. Hence, in terms of the metrics of interest, after the electrical partition, each MG will probably reach different steady-state equilibrium points.

\section{B. Steady-state analysis}

When partitions occur, the characterization of the MG steady-state values for active power, correction term and frequency, $P(\infty), \Delta(\infty)$ and $\Omega(\infty)$ respectively, is obtained from the solution of the equation describing the closed-loop dynamics (17) (when the perturbation is omitted) as $t \rightarrow \infty$.

The focus is first put on $P(\infty)$ and $\Delta(\infty)$, thus using the first two vector-rows of the closed-loop dynamics (17), namely reduced closed-loop dynamics, written as

$$
\left[\begin{array}{c}
\dot{P}(t) \\
\dot{\Delta}(t)
\end{array}\right]=S^{\prime}\left[\begin{array}{l}
P(t) \\
\Delta(t)
\end{array}\right]+U^{\prime} \Omega_{0},
$$

where $S^{\prime} \in \mathbb{R}^{2 n \times 2 n}$ and $U^{\prime} \in \mathbb{R}^{2 n \times n}$ are

$$
S^{\prime}=\left[\begin{array}{cc}
-M v^{2} B & v^{2} B \\
K M & -K\left(\frac{1}{n} C L+\boldsymbol{I}_{n}\right)
\end{array}\right], U^{\prime}=\left[\begin{array}{c}
v^{2} B \\
\mathbf{0}_{n \times n}
\end{array}\right] .
$$

By assuming that all set-point frequencies are the same, $\omega_{0 i}=\omega_{0 j}$, the contribution of the input is in the reduced dynamics (22) satisfies that $U^{\prime} \Omega_{0}=v^{2} B \Omega_{0}=0$ (remind that $B$ (3) is a Laplacian matrix which implies that $B \Omega_{0}=0$ ). Hence, the reduced dynamics (22) simplify to the contribution of the reduced closed-loop system matrix $S^{\prime}$ given in (23) whose solution in known [37]. In fact, being $\lambda_{i}$ and $\mathcal{V}_{\lambda_{i}}$ the $2 n$ eigenvalues and eigenvectors of $S^{\prime}$ respectively, and by using the eigen-decomposition of $S^{\prime}$ as $S^{\prime}=\mathcal{V}_{\lambda} S_{\lambda}^{\prime} \mathcal{V}_{\lambda}^{-1}$ where $S_{\lambda}^{\prime}$ is the diagonal matrix of eigenvalues, the solution of the reduced closed-loop dynamics (22) with $U^{\prime} \Omega_{0}=0$ reduces to

$$
\left[\begin{array}{l}
P(t) \\
\Delta(t)
\end{array}\right]=\mathcal{V}_{\lambda} e^{S_{\lambda}^{\prime} t} \mathcal{V}_{\lambda}^{-1}\left[\begin{array}{l}
P(0) \\
\Delta(0)
\end{array}\right]
$$

Noting that $\lim _{t \rightarrow \infty} e^{S_{\lambda}^{\prime} t}$ is a diagonal matrix with $1 \mathrm{~s}$ in the first $k$ diagonal entries (corresponding to the $k$ zero eigenvalues generated by the electrical and communication Laplacian matrices $B(3)$ and $L(10)$ ) and 0 s in the remaining $2 n-k$ diagonal entries (corresponding to the $2 n-k$ eigenvalues with negative real part), it follows that $\lim _{t \rightarrow \infty} \mathcal{V}_{\lambda} e^{S_{\lambda}^{\prime} t}=\mathcal{V}_{\lambda=0}$ Hence, the solution (24) as $t \rightarrow \infty$ leads to

$$
\left[\begin{array}{l}
P(\infty) \\
\Delta(\infty)
\end{array}\right]=\mathcal{V}_{\lambda=0} \mathcal{V}_{\lambda}^{-1}\left[\begin{array}{l}
P(0) \\
\Delta(0)
\end{array}\right]
$$

It is important to note that the computation of $P(\infty)$ and $\Delta(\infty)$ requires specifying $P(0)$ in $(25)$ in such a way that must be consistent with the MG structure given by (2).

For the steady-state value of the frequency, the expression of $\dot{\Omega}(t)$ given in (17) when $t \rightarrow \infty$ under the assumption that all set-point frequencies are the same, $\omega_{0 i}=\omega_{0 j}$ (and reminding that $B(3)$ is a Laplacian matrix) becomes

$0=\left[\begin{array}{lll}M^{2} v^{2} B & -M v^{2} B-\frac{1}{n} K C L & -K\end{array}\right]\left[\begin{array}{c}P(\infty) \\ \Delta(\infty) \\ \Omega(\infty)\end{array}\right]+K \Omega_{0}$

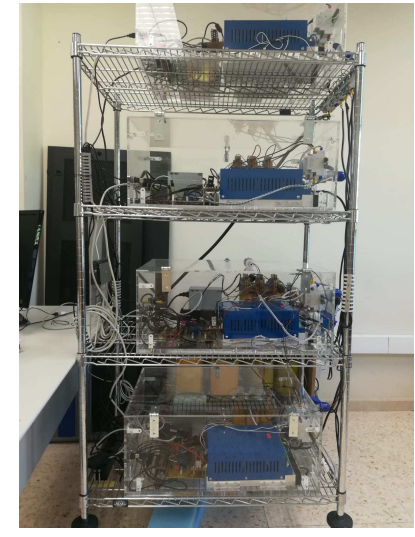

(a) 4-VSI structure.

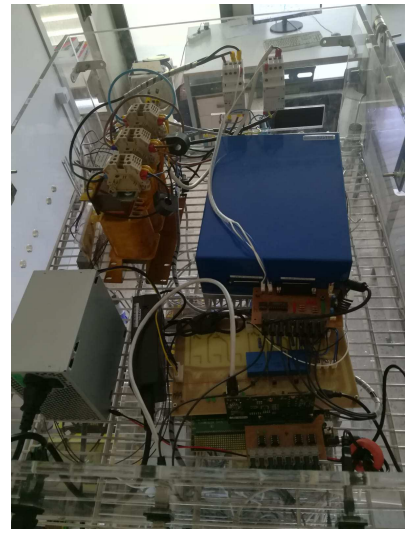

(b) VSI and control hardware.
Fig. 3: Laboratory microgrid.

TABLE I: Nominal values of the laboratory MG components

\begin{tabular}{cll}
\hline \hline Symbol & Description & nominal value \\
\hline$v$ & Grid voltage (rms line-to-line) & $\sqrt{3} 110 \mathrm{~V}$ \\
$\omega_{0}$ & Grid frequency at no load & $2 \pi 60 \mathrm{rad} / \mathrm{s}$ \\
$Z_{1}$ & Line impedance 1 & $0.75 \Omega @ 90^{\circ}$ \\
$Z_{2}$ & Line impedance 2 & $0.30 \Omega @ 90^{\circ}$ \\
$Z_{3}$ & Line impedance 3 & $0.30 \Omega @ 90^{\circ}$ \\
$T_{1}$ & Transformer impedance & $0.62 \Omega @ 37.01^{\circ}$ \\
$T_{2}$ & Transformer impedance & $0.62 \Omega @ 37.01^{\circ}$ \\
$T_{3}$ & Transformer impedance & $1.31 \Omega @ 9.87^{\circ}$ \\
$T_{4}$ & Transformer impedance & $1.31 \Omega @ 9.87^{\circ}$ \\
$Z_{v}$ & Virtual impedance & $3.76 \Omega @ 90^{\circ}$ \\
$Z_{G}$ & Global load impedance & $22 \Omega @ 0^{\circ}$ \\
$P_{G}$ & Global load power & $1.5 \mathrm{~kW}$ \\
$Z_{L 1, L 2}$ & Local load impedances & $88 \Omega @ 0^{\circ}$ \\
$P_{L 1, L 2}$ & Local load powers & $0.5 \mathrm{~kW}$ \\
$m_{i}$ & Gain of the frequency droop & $1 \mathrm{mrad} /(\mathrm{Ws})$ \\
$n_{i}$ & Gain of the voltage droop & $0.5 \mathrm{mV} /(\mathrm{VAr})$ \\
$c_{i}$ & Proportional gain of consensus & 5 \\
$k_{i}$ & Integral gain of consensus & $4 \mathrm{rad} / \mathrm{s}$ \\
\hline \hline
\end{tabular}

By isolating $\Omega(\infty)$ from (26), the steady-state value of the MG frequency is

$\Omega(\infty)=K^{-1}\left[M^{2} v^{2} B-M v^{2} B-\frac{1}{n} K C L \quad K\right]\left[\begin{array}{c}P(\infty) \\ \Delta(\infty) \\ \Omega_{0}\end{array}\right]$

where the equilibrium points for active power $P(\infty)$ and correction term $\Delta(\infty)$ are given in (25).

\section{RESUlts}

\section{A. Laboratory $M G$}

The laboratory MG shown in Figure 3 follows the scheme given in Figure 4. It is composed by four generators $G_{1,2,3,4}$ interfaced by VSI organized in four shelfs, see Sub-figure 3(a), each one containing a full-bridge IGBT inverter and the control and sensing boards, see Sub-figure 3(b). The MG feeds a global load with impedance $Z_{G}$ and two local loads with impedance $Z_{L 1}$ and $Z_{L 2}$. Each power converter is driven by a dual core digital signal processor (32-bit ConcertoF28M36P63C DSP) to program the control strategy (4)-(6) with virtual impedance $Z_{v}$ plus voltage droop control [5] characterized by the proportional control gain, named $n_{i}$. The latter 


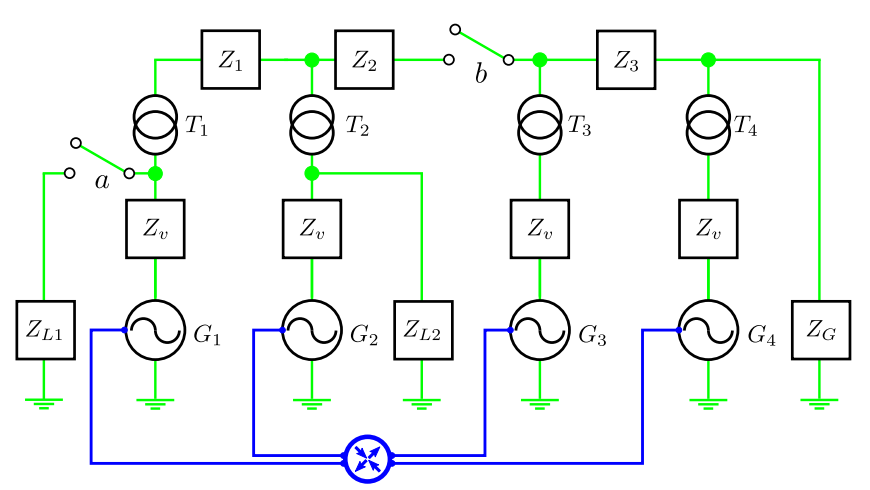

Fig. 4: Microgrid scheme

ensures that voltages amplitudes and the values of the injected reactive power at each VSI will lie within admissible ranges. Future work should assess the effect that partitions have in voltage amplitudes and injected reactive power whenever a reactive power sharing policy [38] is implemented. Hence, the results discussed next omit showing voltage amplitudes and reactive power dynamics because they do not contribute in the analysis presented in this paper.

The MG uses the User Datagram Protocol over a switched Ethernet to allow communication among the four inverters. The diagram also includes both line impedances $Z_{1,2,3}$ modeling the parasitic elements of the cables and isolation transformers $T_{1,2,3,4}$ connected at the output of each inverter. The nominal values of the MG components and control parameters are listed in Table I. The scheme includes two interruptors $a$ and $b$ in the form of electronic relays governed by a digital card. The first one is used to connect or disconnect the local load $Z_{L 1}$ while $b$ allows the electrical partitioning of the MG . The communication partitioning is performed at the Ethernet switch by disabling specific communication ports (drawn as a circle in the bottom part of the figure).

Figure 5 provides a complementary view of the laboratory MG in terms of graph connectivity after the Kron reduction. For each Sub-figure, the top graph corresponds to the electrical connectivity between the four generators which is characterized by the Laplacian matrix $B$ (3). And the bottom graph corresponds to the communication connectivity involving also the four generators, which is characterized by the Laplacian matrix $L$ (10). Thick solid lines between pairs of generators in both graphs represent the existence of connectivity. Thin dashed lines exists only for the clarity of the drawing. Each Laplacian matrix depends on two states $x \mid y$ with the following meaning: 1|1 codifies that no partitions occur (all generators are electrically connected or communicate between them), 1|0 codifies a partition isolating $G_{1}-G_{2}$ from $G_{3}-G_{4}$, and $0 \mid 1$ codifies a partition isolating $G_{1}-G_{3}$ from $G_{2}-G_{4}$ (code $0 \mid 0$ is not used). The particular scenario shown in Sub-figure 5(a) represents the starting operational case where no partitions exists. Sub-figure 5(b) illustrates the case where a partition in the communication graph occurs, Sub-figure 5 (c) is the case where a partition in the electrical graph occurs, and Subfigure $5(\mathrm{~d})$ is the case where a partition in both graph occurs.

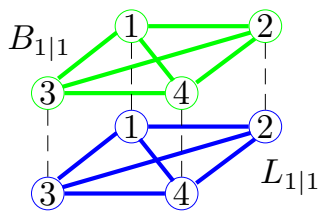

(a) Without partitions

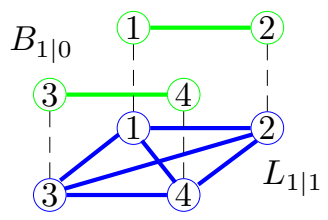

(c) Electrical partition

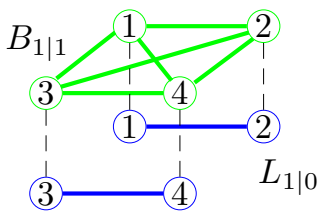

(b) Communication partition

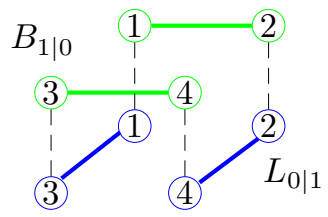

(d) Both with partitions
Fig. 5: MG graph connectivity scenarios.

\section{B. Numerical example}

A numerical example using the laboratory MG (Figure 4) and the control parameters listed in Table I is developed to illustrate the main results given in Section III.

In order to observe the effect of partitions on the closedloop dynamics (17) in terms of the number and role of the 0 eigenvalues of the system matrix $S$ (18), $S$ as well as the input matrix $U$ (19) must be computed. Their values will depend on electrical $B$ (3) and the communication $L$ (10) Laplacian matrices, that also vary according to a particular partition scenario.

When no partitions occur, which is the case of Subfigure 5(a), the Laplacian matrices $B_{1 \mid 1}$ and $L_{1 \mid 1}$ that apply are

$\left(\begin{array}{rrrr}0.155 & -0.056 & -0.051 & -0.047 \\ -0.056 & 0.173 & -0.060 & -0.056 \\ -0.051 & -0.060 & 0.178 & -0.066 \\ -0.047 & -0.056 & -0.066 & 0.170\end{array}\right),\left(\begin{array}{rrrr}3 & -1 & -1 & -1 \\ -1 & 3 & -1 & -1 \\ -1 & -1 & 3 & -1 \\ -1 & -1 & -1 & 3\end{array}\right)$.

which leads to an input matrix $U$ and to a system matrix $S_{n p}$ (where subscript $n p$ stands for non-partition), detailed in Appendix B, in (36) and (37). It is easy to verify that within the eigenvalues of $S_{n p}, \lambda\left(S_{n p}\right)=\{-4,-4,-4,-4$, $-25.6,-25.9,-26.03,-4,-5.79,-6.44,-6.75,0\}$, the 0 eigenvalue appears only once. It addition, it can be verified that $\operatorname{rank}\left(S_{n p}\right)=\operatorname{rank}\left(S_{n p} \mid U\right)=11$, due to the taken non-minimal realization of closed-loop dynamics (17), thus illustrating the discussion given at the end of Section II.

For example, when a communication partition (Subfigure 5(b)) occurs, the corresponding Laplacian matrix $L_{1 \mid 0}$ is

$$
\left(\begin{array}{rrrr}
1 & -1 & 0 & 0 \\
-1 & 1 & 0 & 0 \\
0 & 0 & 1 & -1 \\
0 & 0 & -1 & 1
\end{array}\right)
$$

which leads to a MG characterized by the same input matrix $U$ than before (36) because it does not depend on the communication Laplacian matrix $L$, but with a different system matrix $S_{c p}$ (where subscript $c p$ stand for communication-partition) given also in Appendix B, in (38). It is easy to verify that within the eigenvalues of $S_{c p}, \lambda\left(S_{c p}\right)=\{-4,-4,-4,-4$, 
TABLE II: $P(\infty) \mathrm{kW} / \Delta(\infty) / \Omega(\infty) \mathrm{Hz}$ without perturbation

\begin{tabular}{cccc}
\hline \hline $\begin{array}{c}\text { Initial } \\
\text { condition }\end{array}$ & $\begin{array}{c}\text { No partitions } \\
B_{1 \mid 1} \& L_{1 \mid 1}\end{array}$ & $\begin{array}{c}\text { Comm. partition } \\
B_{1 \mid 1} \& L_{1 \mid 0}\end{array}$ & $\begin{array}{c}\text { Electrical partition } \\
B_{1 \mid 0} \& L_{1 \mid 1}\end{array}$ \\
\hline $597.25 / 0 / 60$ & $597.25 / 0.5972 / 60$ & $597.25 / 0.5972 / 60$ & $449.01 / 0.5722 / 60.12$ \\
$597.25 / 0 / 60$ & $597.25 / 0.5972 / 60$ & $597.25 / 0.5972 / 60$ & $449.01 / 0.5722 / 60.12$ \\
$597.25 / 0 / 60$ & $597.25 / 0.5972 / 60$ & $597.25 / 0.5972 / 60$ & $744.55 / 0.6214 / 59.87$ \\
$597.25 / 0 / 60$ & $597.25 / 0.5972 / 60$ & $597.25 / 0.5972 / 60$ & $744.55 / 0.6214 / 59.87$ \\
\hline $555.88 / 0 / 60$ & $597.25 / 0.5972 / 60$ & $589.16 / 0.5892 / 60$ & $449.01 / 0.5722 / 60.12$ \\
$593.54 / 0 / 60$ & $597.25 / 0.5972 / 60$ & $589.16 / 0.5892 / 60$ & $449.01 / 0.5722 / 60.12$ \\
$588.05 / 0 / 60$ & $597.25 / 0.5972 / 60$ & $605.34 / 0.6053 / 60$ & $744.55 / 0.6214 / 59.87$ \\
$651.53 / 0 / 60$ & $597.25 / 0.5972 / 60$ & $605.34 / 0.6053 / 60$ & $744.55 / 0.6214 / 59.87$ \\
\hline \hline
\end{tabular}

TABLE III: $P(\infty) \mathrm{kW} / \Delta(\infty) / \Omega(\infty) \mathrm{Hz}$ with perturbation

\begin{tabular}{cccc}
\hline \hline Initial & No partitions & Comm. partition & Electrical partition \\
condition & $B_{1 \mid 1} \& L_{1 \mid 1}$ & $B_{1 \mid 1} \& L_{1 \mid 0}$ & $B_{1 \mid 0} \& L_{1 \mid 1}$ \\
\hline $597.25 / 0 / 60$ & $594.06 / 0.5843 / 59.990$ & $+\infty /+\infty / 59.992$ & $447.94 / 0.5595 / 60.11$ \\
$597.25 / 0 / 60$ & $596.18 / 0.5827 / 59.996$ & $+\infty /+\infty / 59.998$ & $450.08 / 0.5580 / 60.12$ \\
$597.25 / 0 / 60$ & $598.31 / 0.5812 / 60.003$ & $-\infty /-\infty / 60.001$ & $743.47 / 0.6050 / 59.88$ \\
$597.25 / 0 / 60$ & $600.45 / 0.5796 / 60.009$ & $-\infty /-\infty / 60.007$ & $745.62 / 0.6035 / 59.89$ \\
\hline \hline
\end{tabular}

$-17.43,-17.83,-11.80,-4.88,-4.59,-4,0,0\}$, the 0 eigenvalue appears twice, hence corroborating Theorem 1 in Appendix A that states that a communication partition adds an additional 0 eigenvalue to the system matrix. In addition, it can be verified that $\operatorname{rank}\left(S_{c p}\right)=10 \neq \operatorname{rank}\left(S_{c p} \mid U\right)=11$, hence the additional 0 eigenvalue acts as an integrator of each input/output map of the closed-loop dynamics (17), thus corroborating the theoretical predictions given in Subsection III-A.

In order to corroborate the theoretical results presented in Sub-section III-B, Table II summarizes the steady-state active power, correction term and frequency values given in (25) and (27) for two different set of initial conditions when the effect of perturbations is not considered $(\Phi(t)=0$ in the closed-loop dynamics (17)) and the input set-point frequencies are the same, $\omega_{0 i}=\omega_{0 j}=60 \mathrm{~Hz}$. Each row corresponds to an inverter with a given initial condition $P(0)$ $\mathrm{kW} / \Delta(0) / \Omega(0) \mathrm{Hz}$ for the scenarios with no partition (Sub-figure 5(a)), communication partition (Sub-figure 5(b)) and electrical partition (Sub-figure 5(c)). The first four rows illustrate the case of equal initial conditions corresponding to a closed-loop equilibrium state. In this case, the power and frequency values only change when an electrical partition occurs (last column) because two electrically isolated sub-microgrids that can not exchange power between them are created, and generators $G_{1}-G_{2}$ feed equally only the local loads while generators $G_{3}-G_{4}$ feed also equally the global load. In addition, generators run in pairs at different frequencies because they reach different steady-state active power values (determined by (5)). When a communication partition occurs nothing is altered and the system remains at the same equilibrium point. The last four rows illustrate the case of different initial conditions corresponding to an open-loop equilibrium state. Under this situation, when no partitions occur, power sharing and frequency regulation is achieved as expected by the control. When a communication partition occurs, two control algorithms run in parallel (one for $G_{1}-G_{2}$ and the other for $G_{3}-G_{4}$ ). By reminding that the communication partition implies adding an integrator to the closed-loop system matrix $S$ that in terms of dynamics affects each input/output relation, the active power and frequency are driven to specific equilibrium points depending on the initial conditions. When an electrical partition occurs (last column), the same scenario illustrated in the first four rows is repeated.

Table III complements the previous example with simulated values for the steady-state active power, correction term, and frequency for the case of equal initial conditions and equal input set-point frequencies, $\omega_{0 i}=\omega_{0 j}=60 \mathrm{~Hz}$ (like the case of the first four rows of Table II) but considering also a constant perturbation $\Phi(t)=\left[\begin{array}{llll}0 & 0.0001 & 0.0002 & 0.0003\end{array}\right]^{T}$ in the closed-loop dynamics (17). When no partitions occur, power sharing and frequency regulation looses accuracy and diverse particular steady-state values that depend on the perturbation values are reached. The same happens with the electrical partition noting that the limitation in power exchange between the two isolated sub-microgrids affects the final values, that are still different but grouped in pairs. When a communication partition occurs, no consensus is reached and the MG crashes. That is, the two isolated consensus algorithms are not able to reach the control goals because they suffer from the announced problem of parallel integrators working on the same plant. Intuitively, looking at the control algorithm (4)-(6), each perturbation term $\varphi_{i}(t)$ determines a different $\omega_{i}(t)$ in (5), that will provoke a permanent error in the integral term of (6) that does not vanishes and leads the system to instability.

\section{Experimental results}

Figure 6 shows the experimental results obtained in the laboratory MG. In the start-up of the system, every 10s, each one of the four inverters enabled with the control (4)(6) connects to the MG to feed both the global and local loads. As it can be observed, after each connection, active power sharing is achieved while the frequency remains at the desired set-point. At $t=62 \mathrm{~s}$ different partitions occur. And at $t=100 \mathrm{~s}$, the local load $Z_{L 1}$ is disconnected, producing a step change in power demand. Sub-figures 6(a), 6(b), 6(c) and 6(d) correspond to the scenarios schematically illustrated in Sub-figures 5(a), 5(b), 5(c) and 5(d). The plotted scenarios in Figure 6 also coincide with the case illustrated in Table III 

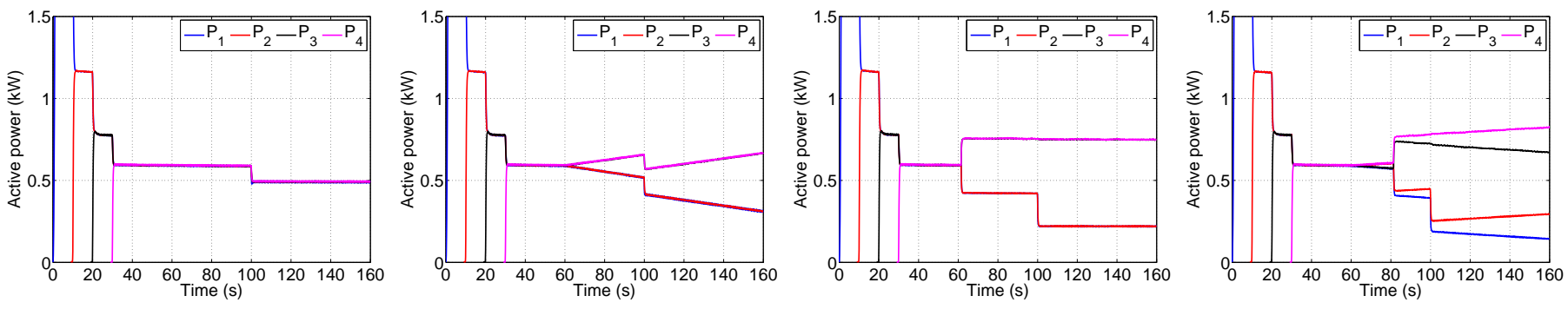

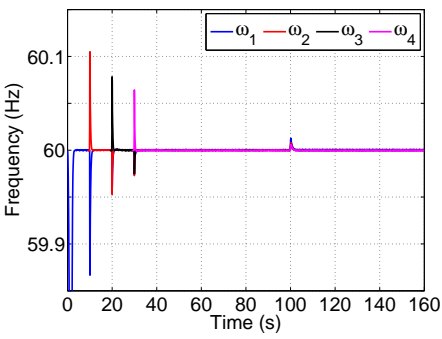

(a) No partitions.

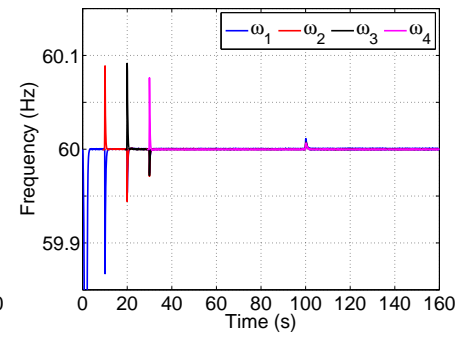

(b) Communication partition.

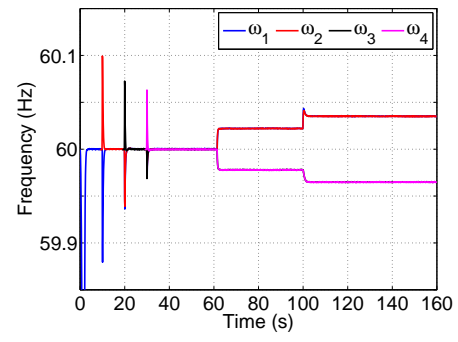

(c) Electrical partition.

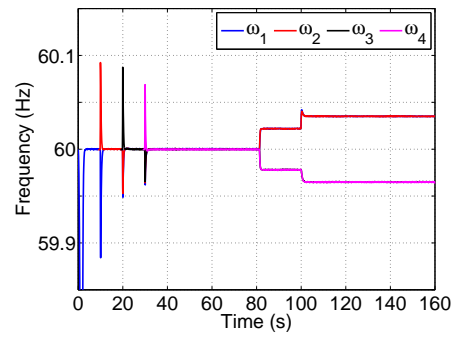

(d) Both partitions.

Fig. 6: Active power and frequency for the 4-nodes MG under the different partition scenarios given in Figure 5

in the sense that initial conditions (that are equilibrium points for the system with no partitions) are the same for all inverters before any partition takes place. In addition, inverters set-point frequencies are the same, $\omega_{0 i}=\omega_{0 j}=60 \mathrm{~Hz}$. Note also that although perturbations are not quantified, they will exist due to e.g. measurement errors when measuring each inverter active power [39], and/or DSPs clock inaccuracies that will affect the operation of the control algorithm [40].

If no partitions occurs (Sub-figure 6(a)), power sharing and frequency regulation is achieved by the control algorithm as expected, coinciding with the numbers shown in Table III. When the local load is disconnected at $t=100 \mathrm{~s}$, the active power abruptly changes but control objectives are satisfied. Note that the effect of the inherent perturbations existing in the physical system can not be observed and do not affect the expected operation, thus being considered here as negligible.

When a communication partition occurs at $t=62 \mathrm{~s}$ leading to the scenario illustrated in Sub-figure 5(b), two control algorithms start acting in parallel as observed in Sub-figure 6(b), one involving $G_{1}-G_{2}$, and the other involving $G_{3}-G_{4}$. And the upper graph corresponding to the active powers shows a slow but unstable dynamics where active powers do not settle. In particular, $P_{1}-P_{2}$ decrease and $P_{3}$ and $P_{4}$ increase, which also coincides with the numbers shown in Table III. The difference between frequencies $\omega_{1}-\omega_{2}$ and $\omega_{3}-\omega_{4}$ can not be appreciated. Hence, the inherent (and distinct) perturbations entering in the system (17), that previously were considered negligible, in this scenario become relevant up to the point that make the dynamics unstable, that is, the $\mathrm{MG}$ crashes. When an electrical partition occurs at $t=62 \mathrm{~s}$ leading to the scenario illustrated in Sub-figure 5(c), two separate MGs start working in parallel as observed in Sub-figure 6(c), one involving $G_{1}-G_{2}$, and the other involving $G_{3}-G_{4}$, but governed by a single control algorithm. In this case, the equilibrium points change in pairs, following the tendency announced by the theoretical results but slightly altered by the perturbations, thus coinciding also with the numbers shown in
Table III. In this case, the difference between pairs of active powers $P_{1}-P_{2}$ and $P_{3}-P_{4}$ (or frequencies $\omega_{1}-\omega_{2}$ and $\left.\omega_{3}-\omega_{4}\right)$ can not be appreciated and the effect of perturbations can be also considered as negligible.

Finally, Sub-figure 6(d) shows the case where both partitions occur leading to the scenario illustrated in Sub-figure 5(d)). The communication disconnection occurs at $t=62 \mathrm{~s}$ and the electrical one at $t=82 \mathrm{~s}$. As it can be observed, the first one provokes the undesired unstable dynamics, while the second one changes the equilibrium points, thus exhibiting a combination of the previous two dynamics shown in Subfigures 6(b) and 6(c).

\section{Discussion}

In modern MGs, electrical and communication networks are becoming the key infrastructures that support the MG power management. This paper has analyzed the impact that failures in these infrastructures has in MG performance, focusing in active power sharing and frequency regulation. The analysis has concentrated on network failures that lead to a situation where islanded sub-networks (called partitions) work in parallel, both in the electrical and communication domain. Figure 6 shows the main results of the analysis. Electrical partitions constraint the energy flows, and electrical subnetworks reach active power sharing and frequency regulation with steadystate values that depend on the load in each partition and the communication scheme, and that differ among partitions. If the newly reached active power values are beyond VSIs rated power, they will trip due to the over-current situation that will occur. Communication partitions constraint the exchange of information required by the frequency regulation task performed by the secondary control, that is, by the consensus control strategy. This impairment leads to unstable dynamics that may imply a cascaded failure of VSI because of the overcurrent situation.

From an engineering point of view, this paper has provided analytical means for understanding the active power and fre- 
quency dynamics whenever failures occur. It also presented the tools for predicting the active power and frequency steady-state values of a failed MG. Moreover, it should permit identifying which management actions should be taken in order to keep the MG in a safe operation scenario whenever failures occur. For example, to avoid the undesirable dynamics given by the experimental case illustrated in Sub-figure 6(b) (or the simulation example given in Figure 2), it seems intuitive that a sub-microgrid that has no communication with another submicrogrid due to a communication failure should be disconnected also at the electrical level to allow both sub-microgrids to work in isolation. Or alternatively, a different approach could be to disable the secondary control until communications are restored. Complementary, in the event of an electrical failure, if the power balance between supply and demand cannot be reached, load shedding policies could be adopted to reduce the risk of collapse. And the trigger for these corrective actions could be based on monitoring the dynamics of the metrics of interest such as VSIs injected active power. All these control strategies should be carefully analyzed and they are left for future work.

\section{CONCLUSions}

This paper has analyzed the effect that communication and electrical partitions have in the performance of islanded MGs governed by a consensus algorithm. A MG closed-loop model has been obtained in terms of the electrical and communication Laplacian matrices that permits characterizing the diverse partition scenarios. The characterization of the MG closed-loop eigenvalues together with the analytical expressions for the steady-state active power and frequency permit identifying the following behaviors. An electrical partition generates isolated sub-MGs, and each one achieves power sharing and frequency regulation with acceptable accuracy under the assumption that the new local supply-demand equilibrium can be reached. A communication partition results in several consensus algorithms working in parallel on the same MG leading to a scenario where power sharing is lost. Care must be taken in this scenario in order to avoid the instability problem. The analysis presented in this paper permits identifying and understanding the performance problems that electrical and communication failures generate in MGs operation. This opens the possibility of designing novel control strategies for mitigating the undesirable behaviors that will otherwise occur.

\section{APPENDIX A}

Next theorems show that for any eigenvector associated to a 0 eigenvalue of the Laplacian matrices $L$ (10) or $B$ (3), it exists a given eigenvector associated to a 0 eigenvalue of the system matrix $S$ (18). This permits concluding that any additional 0 eigenvalue in $L$ or $B$ due to a partition leads to a new 0 eigenvalue in $S$.

Theorem 1. Consider the MG closed-loop dynamics (17) characterized by the system matrix $S$ (18) that depends on the communication Laplacian matrix $L(10)$. Let $\mathcal{N}(S)$ and $\mathcal{N}(L)$ be the null space of $S$ and $L$, respectively. Then

$$
\forall x \in \mathcal{N}(L) \rightarrow \exists \mathcal{V}_{\mathcal{L}}(x) \in \mathcal{N}(S)
$$

where the vector $\mathcal{V}_{\mathcal{L}}(x)$ is

$$
\mathcal{V}_{\mathcal{L}}(x)=\left[\begin{array}{lll}
M^{-1} x & x & 0
\end{array}\right]^{T}
$$

Proof. By computing $S \mathcal{V}_{\mathcal{L}}(x)$ we obtain

$$
S \mathcal{V}_{\mathcal{L}}(x)=\left[\begin{array}{c}
-M v^{2} B M^{-1} x+v^{2} B x \\
K M M^{-1} x-K\left(\frac{1}{n} C L+\boldsymbol{I}_{n}\right) x \\
M^{2} v^{2} B M^{-1} x-\left(M v^{2} B+\frac{1}{n} K C L\right) x
\end{array}\right]_{(30)}
$$

Looking at the first row of (30), and recalling that $M$ is diagonal, it follows that $-v^{2} B x+v^{2} B x=0$. Looking at the second row of (30), and noting that if $x \in \mathcal{N}(L)$ then $L x=0$, it holds that $K x-K \frac{1}{n} C L x-K x=0$. And looking at the third row of (30), by using the fact that $M$ is diagonal and that $x \in \mathcal{N}(L)$, it follows that $M v^{2} B x-M v^{2} B x-\frac{1}{n} K C L x=0$. Hence, it hods that $S \mathcal{V}_{\mathcal{L}}(x)=0$ which implies that $\mathcal{V}_{\mathcal{L}}(x) \in \mathcal{N}(S)$.

Theorem 2. Consider the MG closed-loop dynamics (17) characterized by the system matrix $S$ (18) that depends on the electrical Laplacian matrix $B(3)$. Let $\mathcal{N}(S)$ and $\mathcal{N}(B)$ be the null space of $S$ and $B$, respectively. Then

$$
\forall x \in \mathcal{N}(B) \rightarrow \exists \mathcal{V}_{\mathcal{B}}(x) \in \mathcal{N}(S)
$$

where the vector $\mathcal{V}_{\mathcal{B}}(x)$ is

$$
\mathcal{V}_{\mathcal{B}}(x)=\left[\begin{array}{lll}
x & x & \left(\boldsymbol{I}_{n}-M\right) x
\end{array}\right]^{T}
$$

Proof. By computing $S \mathcal{V}_{\mathcal{B}}(x)$ we obtain

$$
S \mathcal{V}_{\mathcal{B}}(x)=\left[\begin{array}{c}
-M v^{2} B x+v^{2} B x \\
K M x-\frac{1}{n} K C L x-K x \\
M^{2} v^{2} B x-M v^{2} B x-\frac{1}{n} K C L x-K x+K M x
\end{array}\right]
$$

Looking at the first row of (33), and noting that if $x \in \mathcal{N}(B)$ then $B x=0$, it holds that $-M v^{2} B x+v^{2} B x=0$. By applying the same property to the first two terms of the third row of (33), and imposing that it should be zero, the following equality $-\frac{1}{n} K C L x-K x+K M x=0$ should be satisfied, from where it is obtained that

$$
K x=-\frac{1}{n} K C L x+K M x
$$

And substituting (34) in the second row of (33) it holds that

$$
K M x-\frac{1}{n} K C L x+\frac{1}{n} K C L x-K M x=0
$$

which implies that $S \mathcal{V}_{\mathcal{B}}(x)=0$. As a consequence, it holds that $\mathcal{V}_{\mathcal{B}}(x) \in \mathcal{N}(S)$. 


\section{APPENDIX B}

Next, the matrices for the numerical example are presented.

$$
U=\left(\begin{array}{cccc}
5635.0 & -2052.0 & -1858.0 & -1726.0 \\
-2052.0 & 6288.0 & -2196.0 & -2040.0 \\
-1858.0 & -2196.0 & 6457.0 & -2403.0 \\
-1726.0 & -2040.0 & -2403.0 & 6169.0 \\
0 & 0 & 0 & 0 \\
0 & 0 & 0 & 0 \\
0 & 0 & 0 & 0 \\
0 & 0 & 0 & 0 \\
-1.635 & 2.052 & 1.858 & 1.726 \\
2.052 & -2.288 & 2.196 & 2.04 \\
1.858 & 2.196 & -2.457 & 2.403 \\
1.726 & 2.04 & 2.403 & -2.169
\end{array}\right)
$$

\section{REFERENCES}

[1] R. H. Lasseter, "MicroGrids," in Proceedings of the 2002 IEEE Power Engineering Society Winter Meeting, pp. 305-308 vol.1., 2002.

[2] J. A. Lopes, C. L. Moreira, and A. G. Madureira, "Defining control strategies for microgrids islanded operation," IEEE Trans. on Power Systems, vol. 21, no. 2, pp. 916?924, May 2006.

[3] J. Rocabert, A. Luna, F. Blaabjerg and P. Rodrguez, "Control of power converters in AC microgrids," IEEE Trans. on Power Electronics, vol. 27, no. 11 , pp. 4734-4749, Nov. 2012.

[4] J.M. Guerrero, J.C. Vasquez, J. Matas, L.G. de Vicuña, and M. Castilla, "Hierarchical control of droop-controlled AC and DC microgrids: a general approach toward standardization," IEEE Trans. on Industrial Electronics, vol. 58, no. 1, pp. 158-172, Jan. 2011.

[5] M.C. Chandorkar, D.M. Divan, and R. Adapa, "Control of parallel connected inverters in standalone AC supply systems," IEEE Trans. on Industry Applications, vol. 29, no. 1, pp. 136-143, Jan/Feb 1993.

[6] F. Guo, C. Wen, J. Mao, and Y.-D. Song, "Distributed secondary voltage and frequency restoration control of droop-controlled inverter-based microgrids," IEEE Trans. on Industrial Electronics, vol. 62, no. 7, pp. 4355-4364, July 2015.

[7] G. Andersson, P. Donalek., R. Farmer, N. Hatziargyriou, I. Kamwa, P. Kundur, N. Martins, J. Paserba, P. Pourbeik, J. Sanchez-Gasca, R. Schulz, A. Stankovic, C. Taylor, and V. Vittal, "Causes of the 2003 major grid blackouts in North America and Europe and recommended means to improve system dynamic performance," IEEE Trans. Power Syst., vol. 20, no. 4, pp. 1922-1928, Nov. 2005.

[8] Y. Cai, Y. Cao, Y. Li, and T. Huang, "Cascading failure analysis considering interaction between power grids and communication networks," IEEE Trans. Smart Grid, vol. 7, no. 1, pp. 1-9, 2015.

[9] S. M. Rinaldi, J. P. Peerenboom and T. K. Kelly, "Identifying, understanding, and analyzing critical infrastructure interdependencies," IEEE Control Systems, vol. 21, no. 6, pp. 11-25, Dec 2001.

[10] I. A. Tøndel, J. Foros, S. S. Kilskar, P. Hokstad, M. G. Jaatun, "Interdependencies and reliability in the combined ICT and power system: An overview of current research," Applied Computing and Informatics, vol. 14, no. 1, pp. 17-27, 2018.

[11] V. Krishnamurthy and A. Kwasinski, "Effects of power electronics, energy storage, power distribution architecture, and lifeline dependencies on microgrid resiliency during extreme events," IEEE Journal of Emerging and Selected Topics in Power Electronics, vol. 4, no. 4, pp. 1310-1323, Dec. 2016.

[12] B. Falahati and Y. Fu, "Reliability assessment of smart grids considering indirect cyber-power interdependencies," IEEE Trans. Smart Grid, vol. 5, no. 4, pp. 1677-1685, July 2014.

[13] "Current practices in Europe on emergency and restoration", Draft of Network Code on Emergency and Restoration, 2014.

[14] Z. Zhang and M. Y. Chow, "Convergence analysis of the incremental cost consensus algorithm under different communication network topologies in a smart grid," IEEE Trans. Power Systems, vol. 27, no. 4, pp. 17611768,2012

[15] S. Bolognani and S. Zampieri, "A Distributed control strategy for reactive power compensation in smart microgrids," IEEE Trans. on Automatic Control, vol. 58, no. 11, pp. 2818-2833, Nov. 2013.
[16] J. W. Simpson-Porco, F. Dorfler, F. Bullo, "Synchronization and power sharing for droop-controlled inverters in islanded microgrids", Automatica, vol. 49, no. 9, pp. 2603-2611, 2013.

[17] E. Tegling, B. Bamieh and D. F. Gayme, "The price of synchrony: evaluating the resistive losses in synchronizing power networks," IEEE Trans. Control of Network Systems, vol. 2, no. 3, pp. 254-266, Sept. 2015.

[18] L. Y. Lu and C. C. Chu, "Consensus-based droop control synthesis for multiple DICs in isolated micro-grids," in IEEE Transactions on Power Systems, vol. 30, no. 5, pp. 2243-2256, Sept. 2015.

[19] V. Nasirian, Q. Shafiee, J. M. Guerrero, F. L. Lewis and A. Davoudi, "Droop-free distributed control for AC microgrids," IEEE Trans. on Power Electronics, vol. 31, no. 2, pp. 1600-1617, Feb. 2016.

[20] J. Schiffer, T. Seel, J. Raisch and T. Sezi, "Voltage stability and reactive power sharing in inverter-based microgrids with consensus-based distributed voltage control," IEEE Trans. on Control Systems Technology, vol. 24, no. 1, pp. 96-109, Jan. 2016.

[21] J. Schiffer, F. Dorfler, and E. Fridmann, "Robustness of distributed averaging control in power systems: time delays \& dynamic communication topology", Automatica, vol. 80, pp. 261-271, 2017.

[22] J. W. Simpson-Porco, "A theory of solvability for lossless power flow equations - Part I: Fixed-point power flow," IEEE Trans. on Control of Network Systems, vol. PP, no. 99, pp. 1-1, June 2017.

[23] J. W. Simpson-Porco, F. Dorfler and F. Bullo, "Voltage stabilization in microgrids via quadratic droop control," IEEE Trans. on Automatic Control, vol. 62, no. 3, pp. 1239-1253, March 2017.

[24] X. Lu, X. Yu, J. Lai, J. M. Guerrero and H. Zhou, ”Distributed secondary voltage and frequency control for islanded microgrids with uncertain communication links," IEEE Trans. on Industrial Informatics, vol. 13, no. 2, pp. 448-460, April 2017.

[25] N. M. Dehkordi, N. Sadati and M. Hamzeh, "Fully distributed cooperative secondary frequency and voltage control of islanded microgrids," IEEE Trans. Energy Conversion, vol. 32, no. 2, pp. 675-685, June 2017.

[26] A. Pilloni, A. Pisano and E. Usai, "Robust finite-time frequency and voltage restoration of inverter-based microgrids via sliding-mode cooperative control," IEEE Trans. on Industrial Electronics, vol. 65, no. 1, pp. 907-917, Jan. 2018.

[27] Y. Song, D. J. Hill and T. Liu, "Network-based analysis of smalldisturbance angle stability of power systems," in IEEE Transactions on Control of Network Systems, in press, 2018.

[28] F. Dörfler and F. Bullo, "Kron reduction of graphs with applications to electrical networks," IEEE Trans. on Circuits and Systems I: Regular Papers, vol. 1, no. 60, pp. 150?163, 2013.

[29] S.Y. Caliskan, P. Tabuada, "Towards Kron reduction of generalized electrical networks", Automatica, vol. 50, no. 10, pp. 2586-2590, 2014.

[30] P. Kundur, Power System Stability and Control, McGraw-Hill, 1994.

[31] X. Guo, Z. Lu, B. Wang, X. Sun, L. Wang and J. M. Guerrero, "Dynamic phasors-based modeling and stability analysis of droop-controlled inverters for microgrid applications," in IEEE Transactions on Smart Grid, vol. 5, no. 6, pp. 2980-2987, Nov. 2014.

[32] J. Matas, M. Castilla, L. García de Vicuña, J. Miret and J.C. Vasquez, "Virtual impedance loop for droop-controlled single-phase parallel inverters using a second-order general-integrator scheme", Trans. Power Electronics, vol. 25, no. 12, pp. 2993 - 3002, Dec. 2010.

[33] M.C. Pulcherio, A.A. Renjit, M.S. Illindala, A.S. Khalsa, J.H. Eto, D.A. Klapp and R.H. Lasseter, "Evaluation of control methods to prevent collapse of a mixed-source microgrid," in IEEE Transactions on Industry Applications, vol. 52, no. 6, pp. 4566-4576, Nov.-Dec. 2016.

[34] M. Khederzadeh and A. Beiranvand, "Identification and prevention of cascading failures in autonomous microgrid," in IEEE Systems Journal, vol. 12, no. 1, pp. 308-315, March 2018.

[35] C. Godsil and G. Royle. Algebraic Graph Theory. Springer, 2001.

[36] M. Andreasson, D. V. Dimarogonas, H. Sandberg and K. H. Johansson, "Distributed control of networked dynamical systems: Static feedback, integral action and consensus", IEEE Trans. on Automatic Control, vol. 59 , no. 7 , pp. $1750-1764,2014$.

[37] T. Kailath, Linear Systems, Prentice-Hall, New Jersey, U.S.A, 1980.

[38] Y. Han, H. Li, P. Shen, E. A. A. Coelho and J. M. Guerrero, ”Review of active and reactive power sharing strategies in hierarchical controlled microgrids," in IEEE Transactions on Power Electronics, vol. 32, no. 3, pp. 2427-2451, March 2017.

[39] G. Pasini, L. Peretto, and R. Tinarelli, "Study of the accuracy requirements of the instrumentation for efficiency measurements in power conversion systems," IEEE Trans. Instrum. Meas., vol. 62, no. 8, pp. 2154-2160, Aug. 2013

[40] J. Schiffer, C. Hans, T. Kral, R. Ortega, and J. Raisch, "Modeling, analysis, and experimental validation of clock drift effects in low-inertia 


$$
S_{n p}=\left(\begin{array}{cccccccccccc}
-5.635 & 2.052 & 1.858 & 1.726 & 5635 & -2052 & -1858 & -1726 & 0 & 0 & 0 & 0 \\
2.052 & -6.288 & 2.196 & 2.04 & -2052 & 6288 & -2196 & -2040 & 0 & 0 & 0 & 0 \\
1.858 & 2.196 & -6.457 & 2.403 & -1858 & -2196 & 6457 & -2403 & 0 & 0 & 0 & 0 \\
1.726 & 2.04 & 2.403 & -6.169 & -1726 & -2040 & -2403 & 6169 & 0 & 0 & 0 & 0 \\
0.004 & 0 & 0 & 0 & -19.0 & 5.0 & 5.0 & 5.0 & 0 & 0 & 0 & 0 \\
0 & 0.004 & 0 & 0 & 5.0 & -19.0 & 5.0 & 5.0 & 0 & 0 & 0 & 0 \\
0 & 0 & 0.004 & 0 & 5.0 & 5.0 & -19.0 & 5.0 & 0 & 0 & 0 & 0 \\
0 & 0 & 0 & 0.004 & 5.0 & 5.0 & 5.0 & -19.0 & 0 & 0 & 0 & 0 \\
0.0056 & -0.0020 & -0.0018 & -0.0017 & -20.63 & 7.052 & 6.858 & 6.726 & -4.0 & 0 & 0 & 0 \\
-0.0020 & 0.0062 & -0.0021 & -0.0020 & 7.052 & -21.29 & 7.196 & 7.04 & 0 & -4.0 & 0 & 0 \\
-0.0018 & -0.0021 & 0.0064 & -0.0024 & 6.858 & 7.196 & -21.46 & 7.403 & 0 & 0 & -4.0 & 0 \\
-0.0017 & -0.0020 & -0.0024 & 0.0061 & 6.726 & 7.04 & 7.403 & -21.17 & 0 & 0 & 0 & -4.0
\end{array}\right)
$$

power systems," IEEE Trans. on Industrial Electronics, vol. 64, no. 7, pp 5942 - 5951, July 2017.

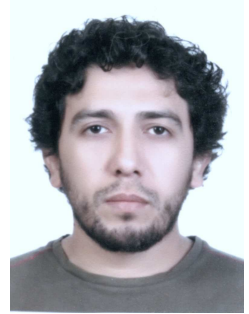

Carlos Xavier Rosero received the B.Sc. degree in automotive electronics engineering from the Army Polytechnic School, Sangolqui, Ecuador in 2008, and the M.Sc. degree in automatic control and robotics from the Technical University of Catalonia (UPC), Barcelona-Spain in 2015. He is currently working toward the Ph.D. degree in automatic control, robotics and vision from UPC. His research interests are centered on real-time control systems and microgrids with emphasis on control strategies.

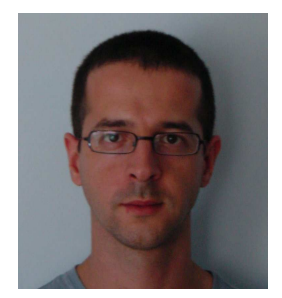

Manel Velasco graduated in maritime engineering in 1999 and received the $\mathrm{PhD}$ degree in automatic control in 2006, both from the Technical University of Catalonia, Barcelona, Spain. Since 2002, he has been an assistant professor in the Department of Automatic Control at the Technical University of Catalonia. His research interests include artificial intelligence, real-time control systems, and collaborative control systems, especially on redundant controllers and multiple controllers with self-interacting systems.

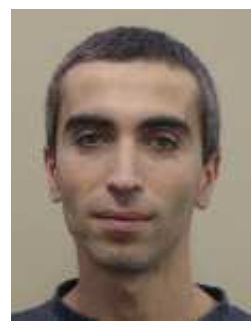

Pau Martí (M'02) received the degree in computer science and the $\mathrm{PhD}$ degree in automatic control from the Technical University of Catalonia, Barcelona, Spain, in 1996 and 2002, respectively. During 2003 he held a research fellow appointment in the Computer Science Department at the University of California at Santa Cruz, US. From 1996 to 2013, he has been an assistant professor in the Department of Automatic Control at the Technical University of Catalonia. Since 2013 he has been an Associate Professor in the Department of Automatic Control at the Technical University of Catalonia. His research interests include embedded and networked control systems, nonlinear control and microgrids.

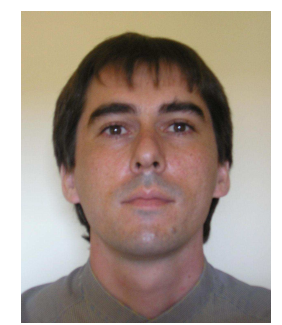

Antonio Camacho received the B.S. degree in chemical engineering, the M.S. degree in automation and industrial electronics, and the Ph.D. degree in electronic engineering, from the Technical University of Catalonia, Barcelona, Spain in 2000, 2009 and 2015 respectively. His research interests include networked and embedded control systems, industrial informatics, and power electronics. 


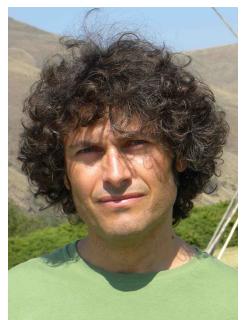

Jaume Miret (M'98) received the B.S. degree in telecommunications, M.S. degree in electronics, and $\mathrm{Ph} . \mathrm{D}$. degree in electronics from the Universitat Politecnica de Catalunya, Barcelona, Spain, in 1992, 1999, and 2005, respectively. From 1993 to 2011, he was an Assistant Professor in the Department of Electronic Engineering, Universitat Politecnica de Catalunya, Spain. Since 2011 he has been an Associate Professor in the Universitat Politecnica de Catalunya, where he teaches courses on digital design and circuit theory. His research interests include dc-to-ac converters, active power filters, and digital control.

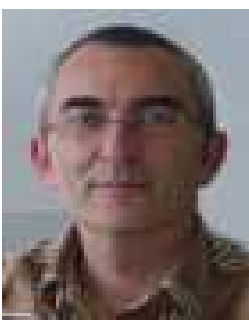

Miguel Castilla received the B.S., M.S. and Ph.D. degrees in telecommunication engineering from the Technical University of Catalonia, Barcelona, Spain, in 1988, 1995, and 1998, respectively. Since 2002, he has been an Associate Professor in the Department of Electronic Engineering, Technical University of Catalonia, where he teaches courses on analog circuits and power electronics. His research interests are in the areas of power electronics, nonlinear control, and renewable energy systems. 